\title{
COACCIÓN Y PESCA ARTESANAL EN EL RÍO PARANÁ. SU IMPACTO EN LAS FAMILIAS PESCADORAS DE BELLA VISTA, CORRIENTES, ARGENTINA
}

\author{
COERCION AND ARTISANAL FISH IN THE PARANÁ RIVER. IT'S IMPACT IN THE \\ FAMILIES THAT FISH AND LIVE IN BELLA VISTA, CORRIENTES, ARGENTINA
}

Nidia Piñeyro'- Agostina Florencia Serial ${ }^{2}$

Fecha de recepción: 29-05-2019.

Fecha de aceptación y versión final: 23-08-2019.

\section{Resumen}

La problemática de la pesca artesanal es compleja. Una de las aristas que identificamos en la investigación en marcha es el carácter conflictivo de la actividad. Dentro de los conflictos detectados hay algunos más visibilizados que otros. En este artículo, desarrollaremos algunas de las consecuencias que tiene en los grupos de pescadores artesanales malloneros de la ribera correntina del Río Paraná la prohibición de pescar en las zonas donde viven. En la Provincia de Corrientes, nos enfocaremos en la localidad de Bella Vista donde la declaración de zona de reserva ictícola por parte de las autoridades provinciales ha dejado en estado de ilegalidad, al menos, a 150 familias que viven exclusivamente de la pesca de especies de gran porte. La permanente exposición a las sanciones (multas y secuestro de artes de pesca) que deviene del carácter ilegal de su práctica productiva también va acompañada de un desprestigio social en sus antiguos espacios de vida y en sus nuevos lugares de trabajo (las riberas de Chaco y Norte de Santa Fe) en los que entran en conflicto con los colegas de otras localidades donde sí está permitido pescar. El objetivo de esta comunicación es discutir algunos indicadores de violencia que aparecen desdibujados cuando se habla de los trabajadores del río en la Región Nordeste de Argentina, entendiendo que la situación de este grupo (vivir en una orilla y pescar en otra) podría ser común a otros colectivos que practican un oficio amenazado. El trabajo de campo (junio de 2013- junio de 2014), el estudio de las reglamentaciones y la ausencia de estos tópicos (coacción, desprestigio, amenaza) en los medios nos hace pensar que estamos asistiendo al desvanecimiento silenciado de una

\footnotetext{
${ }^{1}$ Magíster y Especialista en Desarrollo Social por la FH, Universidad Nacional del Nordeste-UNNE. Profesora en Letras por la misma institución. Doctoranda en Ciencias Sociales y Humanas por la Universidad Nacional de Misiones -UNaM-. Docente Regular responsable de la Cátedra Introducción a las Ciencias Sociales. Coordinadora del Grupo de Investigación y Desarrollo "Estudios Socioculturales del NEA". Integrante del PI "Cultura y turismo en la Región Nordeste de Argentina después del 2000. Un estudio desde la perspectiva de los pobladores locales." Res. 089/19. Miembro académico de la Red WATERLAT-GOBACIT. Domicilio: Dónovan 1550 PB. Resistencia, Chaco, República Argentina. Tel. celular: Tel: 03624 4571850. Correo electrónico: nidiapi@yahoo.com

${ }^{2}$ Profesora en Ciencias de la Educación y estudiante avanzada de la Licenciatura en Ciencias de la Educación, Facultad de Humanidades, UNNE. Miembro de Grupo de Investigación y Desarrollo "Estudios Socioculturales del NEA". Integrante del PI "Cultura y turismo en la Región Nordeste de Argentina después del 2000. Un estudio desde la perspectiva de los pobladores locales." Res. 089/19. Miembro estudiante de la Red WATERLAT-GOBACIT. Domicilio: Av. Edison 1598. Resistencia, Chaco, República Argentina. Tel. Tel: 4573282. Correo electrónico: agostina.serial@hotmail.com
} 
actividad productiva que da trabajo, redes de contención social, contenidos culturales propios a los miembros de las comunidades en que se practica.

Palabras clave: pesca artesanal - reserva ictícola - Río Paraná - conflictos.

Abstract

The problematic situation of artisanal fisheries is complex. One edge that we identified in the ongoing investigation is the controversial nature of the activity. Among the detected conflicts are some more made visible than others. This article will develop the consequences of prohibition to fish in areas where artisanal fishermen off the coast of Parana River Corrientes live. In the province of Corrientes, we will focus on the town of Bella Vista where the declaration of reserve area by the provincial authorities left in a state of illegality at least 150 families who 's only sustenance is fishing for large fish. The permanent exhibition sanctions (fines and seizure of gear) that becomes the unlawful nature of its productive practice also is accompanied by social insecurity in their former living spaces and their new workplaces (the banks of Chaco and northern Santa $\mathrm{Fe}$ ) which conflict with colleagues in other locations where it is permitted to fish. The aim of this paper is to discuss some indicators of violence appear to be blurry when someone refears of workers in the river in the Northeast Region of Argentina understanding that the situation of this group (live on a shore and fishing in another) may be common to other groups that practice a profession threatened. Fieldwork (june, 2013 at june, 2014), the study of regulations and the absence of these topics (coercion, insecurity, threat) in the media makes us think that we are witnessing the fading muted in a productive activity that provides jobs, social safety nets, cultural content. to the members of the communities in which it is practiced.

Key words: artisanal fisheries - reserved area - Parana River - conflict.

\section{Introducción}

En este artículo presentamos algunos de los resultados del trabajo realizado en el marco del proyecto de investigación "El Río Paraná como escenario de conflicto. Actividades productivas, territorialidades y sujetos en las riberas de la Región Nordeste después del 2000". En ese contexto estudiamos escenarios conflictivos, estrategias de ocupación y ámbitos de disputas en el área de influencia de las capitales provinciales de Chaco y Corrientes (Argentina) en la última década.

Hasta la fecha (2018) hemos avanzado en la caracterización y descripción de algunas de las interrelaciones de actividades económicas entre las cuales seleccionamos las vinculadas a la pesca artesanal, especialmente en la localidad de Bella Vista, Provincia de Corrientes, donde la declaración de reserva constituye una restricción que afecta a los trabajadores del río ${ }^{3}$ de una manera contundente y genera conflictos particulares.

\footnotetext{
3 Utilizamos la denominación "trabajador" por oposición a empleo. En el primer caso, la tarea no implica ser un asalariado en relación de dependencia. En cambio, entendemos por empleo un trabajo cuyas características más destacadas son la retribución en forma de salario a cambio de una tarea jornalizada y la relación entre un patrón y una persona que depende económicamente de esta retribución.
}




En otros trabajos hemos expuesto algunas de las condiciones específicas para el desarrollo del oficio en la Región NEA (Nordeste Argentino): restricción de la actividad por múltiples mecanismos políticos y condiciones naturales; tensiones y conflictos intra, inter y extra grupales; judicialización en Chaco y prohibición en casi toda la ribera de Corrientes; fuerte presunción de predación, ilegalidad e ignorancia sobre el grupo por parte de la prensa, organismos del Estado y, la concomitante presión por la reconversión a otras actividades o el desaliento de la reproducción del oficio (Piñeyro, Attías y Lombardo, 2015; Piñeyro y Serial, 2013).

El tema que abordaremos en este caso corresponde a una trama de conflictos donde confluyen problemas inter-grupales (entre trabajadores del río de distintas jurisdicciones provinciales) y extra-grupales (con el Estado y los pescadores deportivos) y configuran, a nuestro entender, casos de violencia.

En este sentido nuestro trabajo se inscribe en la línea de los estudios sociales que consideran la violencia como una relación de poder en la cual quien se sitúa en la posición superior somete a quien está en la posición inferior: "Es un vínculo, una forma de relación social por la cual uno de los términos realiza su poder acumulado" (Izaguirre, 1996 citado por Pratesi, 2014, p. 106).

Seleccionamos una de las localidades de la Provincia de Corrientes en la que, paradójicamente, está prohibida la pesca y están activas más de un centenar de embarcaciones malloneras cuya producción es absorbida en su totalidad por el mercado interno.

El estudio general tiene entre sus objetivos dar cuenta de algunos indicadores de violencia en la relación que involucra a los pescadores malloneros, los deportivos y el Estado a través de sus instituciones.

En este caso, nos circunscribiremos a los resultados del análisis de entrevistas semi-estructuradas realizadas en junio de 2013 y junio de 2014 a pescadores malloneros y deportivos de Bella Vista, Corrientes. Estos datos son interpretados en correlación con otros que emanan del Poder Legislativo y la prensa de un mismo período o de otro inmediatamente anterior.

Estamos conscientes de que, por lo exiguo del corpus y la selección de operaciones de análisis, no agotaremos la complejidad del fenómeno bajo estudio. Sin embargo, la revisión de los antecedentes ${ }^{4}$ nos hace pensar que el nuestro es, aún en estas condiciones, un aporte al conocimiento de grupos productivos en riesgo desde una perspectiva interdisciplinaria.

\footnotetext{
${ }^{4}$ En trabajos reseñados (Wellington Castellucci, 2007; Saavedra Gallo, 2013; Hernández y Sandoval, 2015) encontramos una serie de problemas de coacción comunes que afectan a las comunidades pescadoras. Las mismas pueden sintetizarse así: a) los territorios donde se practica el oficio están en plena transformación; b) la misma exhibe una aceleración en las últimas décadas; c) el cambio del uso del suelo es funcional a la urbanización de estas áreas, lo cual favorece el avance de otros sectores productivos; d) la convergencia de todos o de algunos de estos factores impacta en la vida de los grupos dedicados a la pesca artesanal en diversos aspectos que se expresan en el cambio de sus rutinas de trabajo, en la organización familiar y en las relaciones sociales.
} 


\section{La pesquería correntina. Espacios de conflicto y síntomas de violencia}

La Provincia de Corrientes exhibe una riqueza extraordinaria en materia de agua. Tiene dos cuencas hidrográficas principales formadas por los cauces del Río Paraná y del Río Uruguay. Muchos de los ríos interiores de la provincia desaguan en alguno de ellos. Mientras que el Río Uruguay abraza a Corrientes por el Este, y sus afluentes son el Aguapey, el Miriñay y el Mocoretá -uno de los límites con Entre Ríos-, el Paraná recorre la Provincia de Corrientes por el Oeste y al Norte, y se le suman los Ríos Santa Lucía y Corrientes. A este último se le suman las aguas del Arroyo Batel, y el Río Guayquiraró 5 .

Como ya se adelantó, en este trabajo nos concentramos en la ribera correntina bañada por el Río Paraná. Allí la pesca comercial está prohibida en casi toda su longitud mientras que la pesca deportiva sigue creciendo como atractivo turístico.

En la imagen se puede apreciar el curso del Paraná a la altura de la Provincia de Corrientes y las localidades ribereñas. En rojo, las zonas restringidas o prohibidas para la pesca comercial.

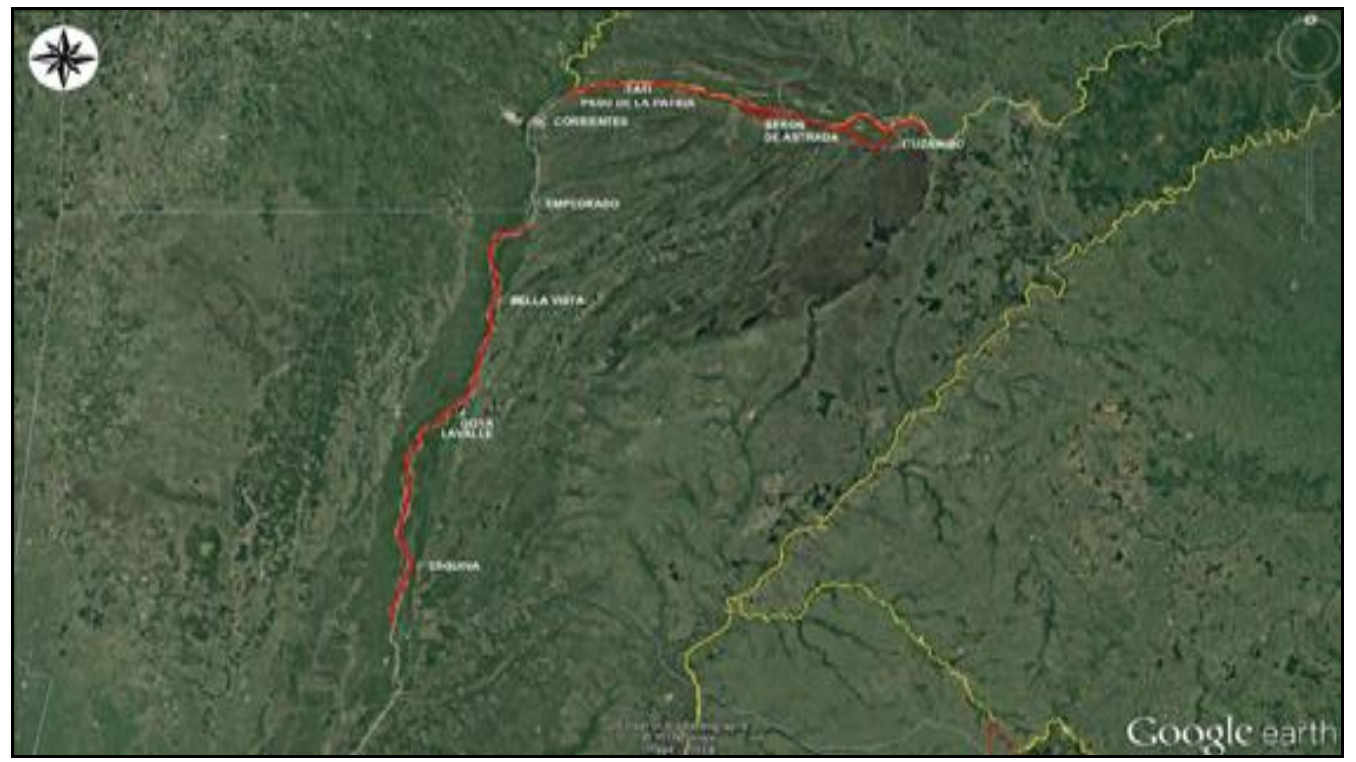

Imagen 1: Zonas de ribera en Corrientes donde está prohibida o restringida la pesca comercial. Fuente: Google Eart. 2016 y reelaboración del equipo (Ricardo Lombardo y Ana María Attías)

La afluencia turística ligada a la pesca excede las fechas de los torneos deportivos nacionales e internacionales (al menos ocho, entre Esquina e Ituzaingó) en los que se extraen peces de gran porte como el dorado (Salminus brasiliensis), el pacú (Piractus mesopotamicus) y el surubí (Pseudoplastytoma corruscans), entre otros altamente demandados en el mercado.

\footnotetext{
${ }^{5}$ Disponible en http://www.corrientes.com.ar/geografia-hidro.htm (Consulta realizada 09/09/2016)
} 
Ligadas también a la industria turística hay actividad hotelera, gastronómica y servicios de embarcaciones con guía para adentrarse en el río desde Semana Santa hasta fin de año, período en que aplica la veda total durante aproximadamente 50 días cuyo objetivo es garantizar la reproducción de algunas especies del río o, al menos, reducir el esfuerzo de pesca.

Las justas deportivas son publicitadas profusamente tanto por el sector privado como por los organismos estatales y tienen como público objetivo a un grupo de cierto nivel adquisitivo disperso en un área geográfica más allá de las fronteras de Argentina. Entre los visitantes más selectos destacan los contingentes brasileros que vienen a Corrientes atraídos por la captura del dorado.

Una búsqueda en Internet como la que sigue "pesca deportiva en Corrientes" arroja como resultado más de 300.000 páginas. Seleccionamos, a modo de ejemplo, un sitio oficial que ofrece un cuadro sintetizador de las fiestas de pesca acompañado con el siguiente texto:

\begin{abstract}
"Pesca en Corrientes
La excepcional presencia del Dorado en las costas de los Ríos Corrientes y Paraná, convierten a la pesca en Corrientes como punto de encuentro de los adeptos más experimentados del mundo. A él se suman el Pacú, Surubí, Patí, Manguruyú y la Boga. Año tras año los pescadores vienen al encuentro de la especie más importante de las aguas correntinas tratando de cumplir el sueño de atrapar a tan extravagante presa. El Dorado, que llega a los 25 kilos, resulta desafiante por su característica de salvaje y luchador". ${ }^{6}$
\end{abstract}

Tabla N 1: "Fiestas de Pesca en Corrientes"

\begin{tabular}{|l|l|l|}
\hline \multicolumn{1}{|c|}{ Ciudad } & \multicolumn{1}{|c|}{ Torneo } & \multicolumn{1}{c|}{ Calendario } \\
\hline Esquina & Fiesta Nacional del Pacú & Marzo \\
\hline Paso de la Patria & Tornero Apertura del Dorado & Marzo-abril \\
\hline Goya & Fiesta Nacional del Surubí & Septiembre \\
\hline Ita Ibaté & $\begin{array}{l}\text { Concurso del Mercosur de Boga y } \\
\text { Pacú }\end{array}$ & Junio \\
\hline Ituzaingó & $\begin{array}{l}\text { Concurso Integración de Pesca } \\
\text { del Surubí }\end{array}$ & Julio \\
\hline Paso de la Patria & Fiesta Nacional del Dorado & Agosto \\
\hline Goya & $\begin{array}{l}\text { Concurso Argentino de Pesca } \\
\text { Variada Embarcada }\end{array}$ & Septiembre \\
\hline Bella Vista & $\begin{array}{l}\text { Concurso de Pesca Variada } \\
\text { Embarcada }\end{array}$ & Noviembre \\
\hline
\end{tabular}

Fuente: Elaboración propia.

En estas localidades donde se promueve la pesca deportiva -y está restringida la comercial- se configura una atmósfera de fricciones múltiples. Los pescadores comerciales no han abandonado el oficio y, de hecho, siguen abasteciendo de pescado a restaurantes y a particulares. Para hacerlo, en Bella Vista, después de la

\footnotetext{
${ }^{6}$ Disponible en: http://www.corrientes.com.ar/pesca-deportiva.htm (Consulta realizada el 19/09/2016)
} 
Declaración de Zona de Reserva por el Decreto Provincial №1970/8937 conviven con una serie de dificultades que desalientan la reproducción de su actividad económica y su particular modo de vida.

La violencia, desde la teoría social de la acumulación (Marx, 1946 y 1968; citado por Pratesi, 2014, p.105) consiste en la desposesión de tiempo, productos, derechos, prestigio y relaciones que se acumulan a favor de otro individuo o grupo.

Los escenarios conflictivos detectados hasta el momento pueden ser clasificados en inter-grupales (con otras comunidades del mismo oficio) y extragrupales (con los pescadores deportivos y organismos del Estado).

En todos los casos es posible encontrar observables de coacción que van desde conductas que atentan contra la reputación del pescador comercial hasta la obstaculización de sus tareas con el fin de que abandone su trabajo.

\subsection{Los conflictos inter - grupales. La migración como obstáculo y desposesión de derechos}

Los observados hasta el momento están en relación con sus vecinos, los pescadores artesanales - comerciales de las riberas del Norte de la Provincia de Santa $\mathrm{Fe}$, lugar que opera como espacio de refugio para afrontar la imposibilidad de trabajar en sus localidades de origen.

En la Provincia de Corrientes, como en la Provincia del Chaco y en la de Santa $\mathrm{Fe}$, los malloneros no constituyen un grupo homogéneo.

Las entrevistas a pescadores de Bella Vista están indicando que los de esa localidad no están asociados -aunque alguna vez intentaron agruparse, sin éxito- y que viven su oficio en situación de extrema precariedad. Los chaqueños tienen razón dicen:

Nosotros aguantamos solos, cada uno por su lado. Nos falta unión. Cuando a uno le sacan las herramientas o lo que pesca, no hacemos nada. Perdemos todo y empezamos de cero. [Bella Vista, junio de 2013].

Este comportamiento podría comprenderse mejor si imaginamos una escala de prioridades donde la zonificación impuesta es el problema más grave para las familias que viven exclusivamente de la pesca.

Cuando se vive en un lugar y se debe pescar en otro se presenta un problema extra. Quienes viven en la orilla correntina del Sur (Esquina, Lavalle, Goya y Bella Vista) y se aventuran al ejercicio del oficio en la Provincia de Santa Fe, cargan con una nueva exigencia, la de acreditar allí su residencia como requisito para trabajar. La reserva, dicen los pescadores comerciales, es como una veda permanente.

\footnotetext{
7 Decreto Provincial №1970/89: se declara Zona de Reserva el tramo del Río Paraná comprendido entre la boca del Arroyo Izoró al Sur y la desembocadura del Arroyo de Ambrosio al Norte, desde tierra firme hasta el límite interprovincial de las aguas, quedando prohibida en esa zona la pesca con elemento mallón, red o tramallo. http://www.nuestromar.org/adobe/Marco regulario Pesca Continental Argentina.pdf
} 
Estamos obligados a trabajar escondidos, a ser delincuentes, teniendo el río en nuestras narices. Somos ilegales acá y ahora también en Santa Fe. [Bella Vista, junio de 2013].

En las orillas de Bella Vista hay más de 100 embarcaciones malloneras que migran a las orillas santafesinas con el riesgo permanente de que sus dueños sean multados 0 pierdan sus herramientas de trabajo.

El mallonero en esta zona digamos que no existe, hay que pescar en Santa Fe [...] Nos domina la presión que hay hacia nosotros, los de fauna [por los empleados de la Dirección de Fauna, agentes que tienen a cargo el control] cada vez nos persiguen más, andamos como delincuentes, y tenemos que dejar cuando hay inspección. [Bella Vista, junio de 2014].

Al padecimiento permanente de los efectos de la regulación impuesta por el Estado hay que agregarle otra consecuencia negativa, el aislamiento social. No pueden ejercer su trabajo en su lugar, pero tampoco son bienvenidos en otra jurisdicción donde sus colegas sí pueden hacerlo.

Para ser legales hay que ir a otro lado... en Santa Fe podemos tener el carnet después de dos años de tener el domicilio [...] no hay problema, pero vivimos en infracción porque no tenemos carnet, no nos reconocen nuestro oficio. [Bella Vista, junio de 2014].

Esta indiferencia del gobierno es interpretada como parte de una estrategia oficial para terminar con la pesca comercial en la zona. La conclusión sobre la violencia de la que son víctima surge con claridad:

Al pescador comercial el gobierno lo quiere sacar del río... nos tildan de depredadores, cuatreros.

En la Imagen 2, la localización del Tramo Sur (Tramo 1) donde se señalan en celeste los estados provinciales que comparten las aguas del Paraná y la situación de Bella Vista, frente a la Provincia de Santa Fe. 


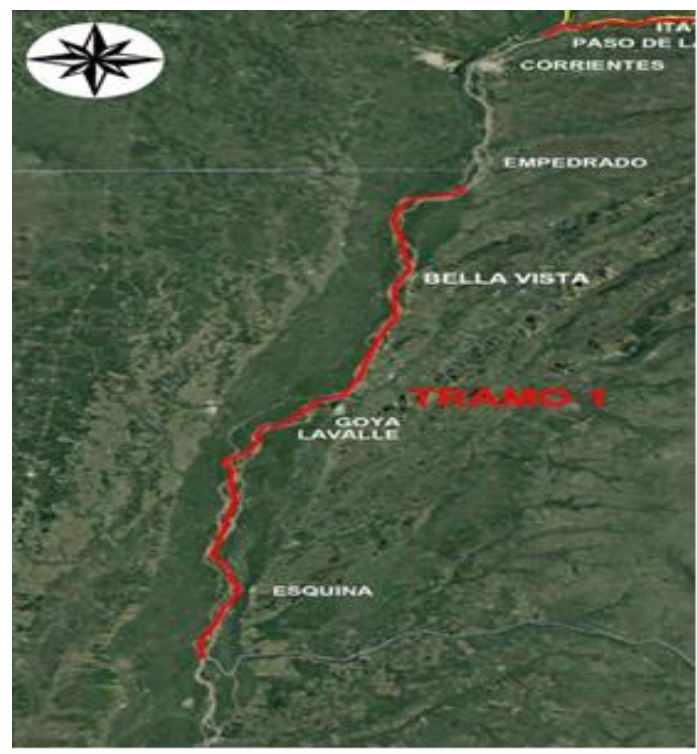

Imagen 2: Zonas de ribera de aguas compartidas entre Corrientes, Chaco y Santa Fe y situación de Bella Vista.

Fuente: Google Eart. 2016 y reelaboración del equipo (Ricardo Lombardo y Ana María Attías).

\subsection{Los conflictos extra grupales. La negación de la dignidad y la autonomía}

En las entrevistas realizadas a los pescadores comerciales, además de las atribuciones de abandono y persecución del gobierno, se tematiza con frecuencia la comparación que hacen de su situación con la de los pescadores deportivos.

Los deportivos vienen y hacen su deporte, llevan pescado y dicen que lo largan, hacen su pesca con devolución. [...]La diferencia con los deportivos es que nosotros vivimos del pescado... si no sacás pescado hoy, mañana no comés [...] Acá es reserva para el pescador comercial, pero para el deportivo no... tiene que ser equitativo y respetaremos [...] "la ilegalidad nos margina. [Bella Vista, junio 2014].

Por su parte, los deportivos atribuyen como rasgo distintivo de los comerciales la falta de cuidado de la fauna. El presidente de la Asociación de Pescadores Deportivos de Bella Vista afirma al ser entrevistado:

No sirve la reserva porque [...] el pescado migra [...] pero si ponés una malla no hay escapatoria para esos peces [...] Todo lo que cae en la malla lo levantan. [refiriéndose a los pescadores comerciales]. [Bella Vista, junio 2013].

Respecto de la complicada situación de los malloneros nuestro entrevistado manifiesta que es una opción de ellos y que es la falta de visión lo que les impide integrarse a la economía local de otra forma. Sostiene que la pesca deportiva moviliza muchos puestos de trabajo y dinamiza la economía de la región donde se la practica:

Los pescadores pueden ser reconvertidos, los mejores guías de pesca son aquellos que son llevados por los pescadores comerciales... Hay que transformar esa pesca en un servicio. [...] Sabe Usted ¿cuánto están dispuestos a pagar los turistas extranjeros por un día de pesca en el Tierra del Fuego? [Bella Vista, junio 2013]. 
Los malloneros dicen que la idea de ir a la pesca turística, además de volverlos esclavos de los deportivos, no asegura el cuidado de la fauna ictícola.

El dorado está prohibido para nosotros, pero para el deportivo no... porque dicen que ellos devuelven... Pero más de una vez vimos cómo los turistas de Brasil vienen con un freezer vacío y se vuelven con uno lleno. [Bella Vista, junio 2013].

Cuando el deportivo se refiere a la reconversión de la pesca comercial en un servicio, asume que los mejores conocedores del río son los malloneros. Pero, aunque hay una valorización del saber de estos trabajadores, entiende que

Hay que reeducarlos, especialmente en el trato con la gente [Bella Vista, junio 2013].

Una síntesis apretada de los actos violentos que soportan los malloneros por parte de los deportivos incluye la desvalorización de su oficio por improductivo, una fuerte presunción de predación y de ignorancia. La propuesta de reconvertirlos puede ser interpretada como la intención de desposeerlos de tiempo, productos y relaciones sociales en beneficio de sus eventuales empleadores. Los comerciales de Bella Vista, a su vez, construyen la imagen del deportivo con los siguientes rasgos: mendacidad, privilegio, explotación. Sobre esto último tienen muy claro que estar en el río por su cuenta no es lo mismo que emplearse en una agencia turística:

Actualmente somos nuestros propios patrones, si somos guías, vamos a trabajar para otro. [Bella Vista, Corrientes, junio 2013].

\subsection{Las reglamentaciones en materia de pesca en la Provincia de Corrientes. La acumulación a favor del turismo}

En el caso de Corrientes, la máxima autoridad que regula la actividad es el Ministerio de Producción, Empleo y Turismo. Llamamos la atención sobre la dependencia de Estado porque esto podría facilitar la comprensión de las políticas adoptadas en torno a la pesca deportiva y la pesca comercial. Corrientes ha optado por instalarse como un nicho turístico centrado en la explotación de la belleza ribereña, incluida la pesca deportiva. Esta política de Estado posibilitó la emergencia de una serie de emprendimientos comerciales en torno a los recursos del río: cabañas, pesca con guía, comida regional a base de pescado, recreación de playa, etc.

En materia de áreas de reservas, éstas se establecen para restringir parcial o totalmente la actividad de pesca.

La Ley N $N^{\circ} 3915$ (Reserva de Goya) establece una restricción para proteger la fauna en todas las islas e islotes, ríos, riachos, arroyos y todo curso de agua ubicado al Oeste del Departamento de Goya, prohibiéndose la pesca comercial en dicha zona, según los siguientes límites: NORTE: Boca del Isoró; SUR: Desembocadura del Arroyo Aguarachay;

ESTE: tierra firme del Departamento Goya y al OESTE: Canal divisorio del Río Paraná (Límite Interprovincial con Santa Fe) ${ }^{8}$.

\footnotetext{
${ }^{8}$ Ley No 3915. Disponible en http://www.nuestromar.org/adobe/Marco regulario Pesca Continental Argentina.pdf
} 
En el caso de Goya, es evidente que el Estado ha optado porque la pesca sea un atractivo exclusivo para los turistas. Esto no significa, en términos estrictos, que el objetivo sea proteger la fauna.

Nótese en la Fotografía $N^{\circ} 1$ la identificación de la localidad con la pesca deportiva. Es difícil imaginar que estos deportistas estén preocupados por el cuidado de alguna especie. Sin embargo, es la imagen de otra página oficial de la Provincia de Corrientes que aparece con la búsqueda "cuál es la distancia desde Corrientes a Goya" 9.

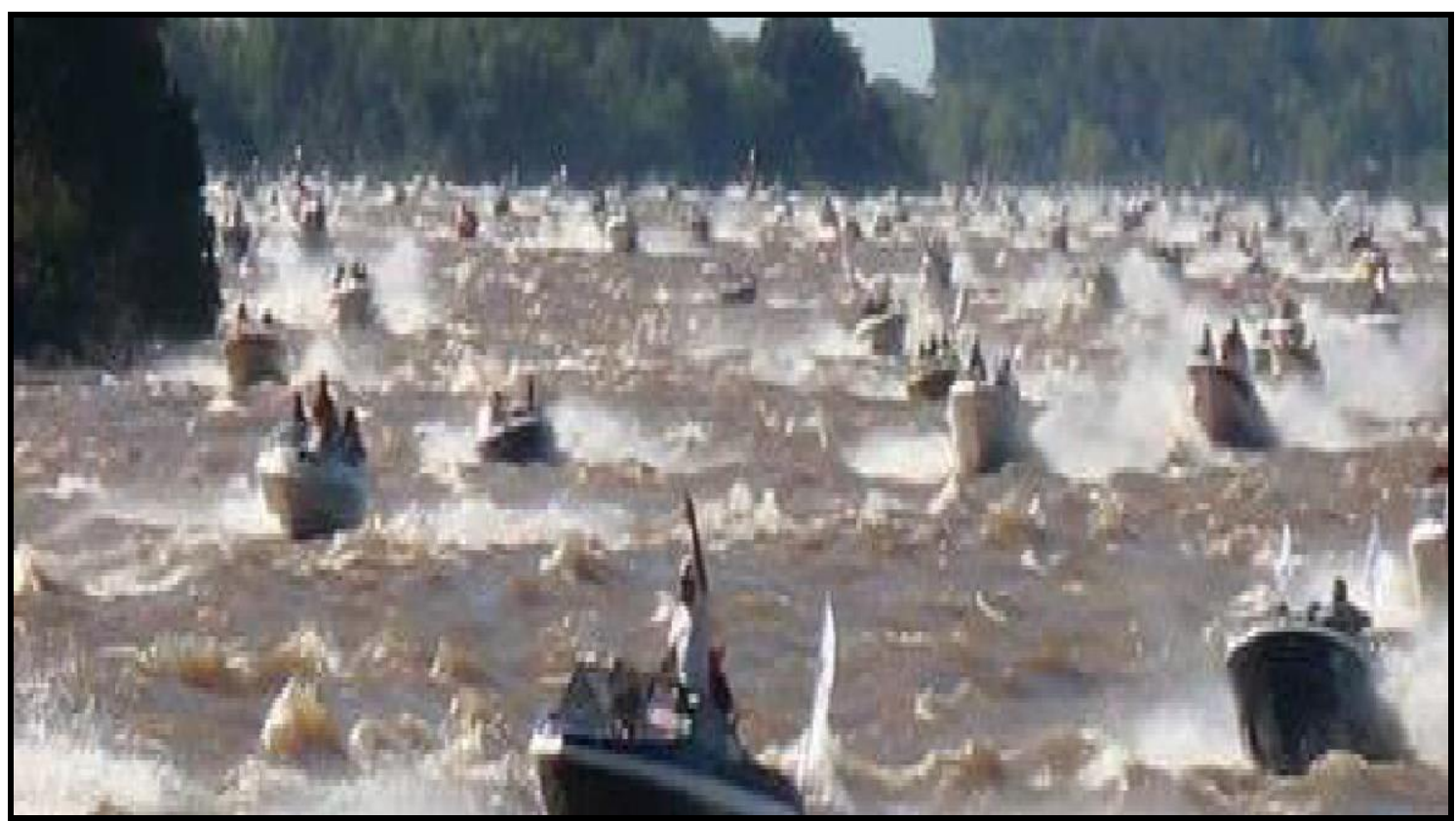

Fotografía $\mathrm{N}^{\circ} 1$ : Imagen de Goya publicitada en la Página oficial de Turismo de la Provincia de Corrientes donde se asocia la localidad a la pesca deportiva.

Fuente: Página oficial de Turismo de la Provincia de Corrientes.

También la Disposición de Dirección de Fauna N 190/87 prohíbe la pesca comercial en el tramo del Río Paraná que va desde el km 1250 hasta el km 1455 (Extremo Oeste de la Reserva de Paso de la Patria hasta Puerto Ituzaingó) ${ }^{10}$.

En el Decreto Provincial N¹970/89 se declara Zona de Reserva el tramo del Río Paraná comprendido entre la boca del Arroyo Izoró al Sur y la desembocadura del Arroyo de Ambrosio al Norte, desde tierra firme hasta el límite interprovincial de las aguas, quedando prohibida en esa zona la pesca con mallón, red o tramallo ${ }^{11}$.

En el caso de la Ley №4827/94 se declaran Zonas de Reserva de fauna íctica en la jurisdicción de aguas territoriales que corresponden a la Provincia de Corrientes,

9 Disponible en http://corrientesturismo.gob.ar/content/men\%C3\%BA-de-mapas-distancia-de-corrientesala-interior (Consulta realizada 21/09/2016).

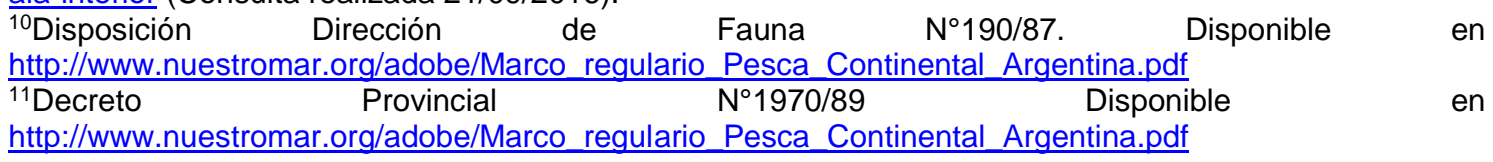

Revista del Instituto de Investigaciones en Educación

ISSN $1853-1393$

Resistencia - Chaco - Argentina 
a los tramos del Río Paraná y sus afluentes que comprenden los Departamentos de Esquina, Goya, Lavalle, Bella Vista (desde el km. 853 hasta el km1126). La misma norma prohíbe en las Zonas de Reserva mencionadas la pesca comercial en cualquier modalidad; así como el desembarco de los productos de las mismas en los puertos o costas de dichos departamentos ${ }^{12}$.

En la Fotografía N² vemos una imagen de la ciudad de Bella Vista. Al fondo, embarcaciones de recreo $\mathrm{y}$, en primer plano, una playa colmada de gente en época estival.

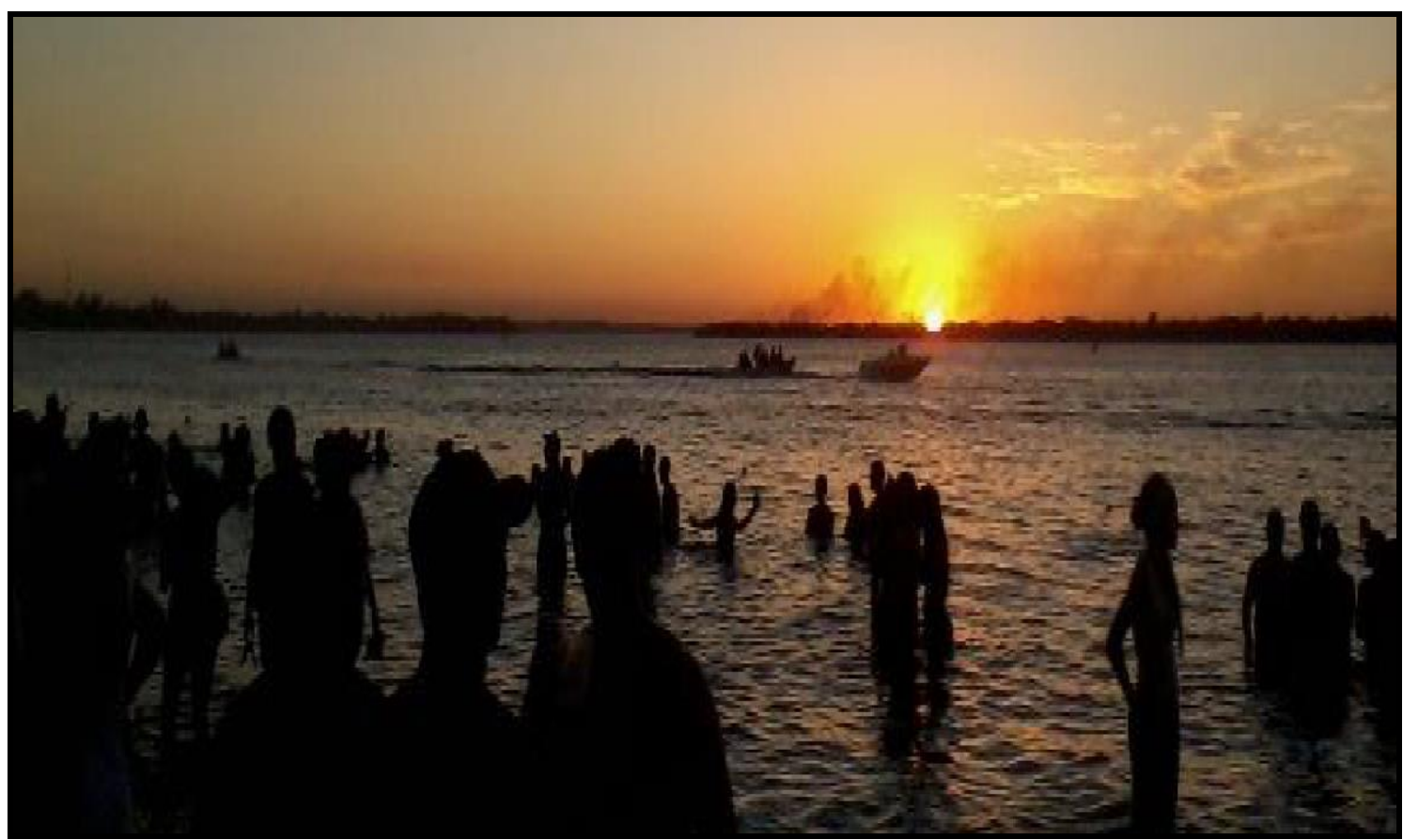

Fotografía $\mathrm{N}^{\circ}$ 2: Imagen de Bella Vista publicitada en la página oficial de Turismo de la Provincia de Corrientes donde se asocia la localidad a las actividades de playa. ${ }^{13}$

Se identifican, además, en la legislación aplicable a la Provincia de Corrientes el Decreto Nacional No 1034/52 (Reserva de Paso de la Patria) y el Decreto Provincial $N^{\circ} 3376 / 57$ - texto según modificación del artículo $1^{\circ}$ del Decreto $N^{\circ} 4190 / 59$ (Reserva de Esquina). En este último caso se establece que la Zona de Reserva para la pesca es la correspondiente al sector compartido entre los siguientes límites: OESTE, margen izquierda de Río Paraná; ESTE, Río Corrientes; NORTE, una línea normal a la boya km 810 del Río Paraná, zona que comprende el Río Corrientes y Riachos de su influencia con el Río Paraná14.

\footnotetext{
${ }^{12}$ Ley

$\mathrm{N}^{\circ} 4827 / 94$

Disponible

en

http://www.nuestromar.org/adobe/Marco regulario Pesca Continental Argentina.pdf

13 Disponible en http://corrientesturismo.gob.ar/content/men\%C3\%BA-de-mapas-distancia-de-corrientesala-interior

14 Decreto Nacional №34/52 y el Decreto Provincial $N^{\circ}$ 3376/57. Disponible en http://www.nuestromar.org/adobe/Marco regulario Pesca Continental Argentina.pdf
}

Revista del Instituto de Investigaciones en Educación ISSN $1853-1393$

Resistencia - Chaco - Argentina 

Provincia.

En la Imagen $\mathrm{N}^{\circ} 3$ se aprecian en rojo las zonas de ribera en el Norte de la



Imagen 3: Zonas prohibidas en el Río Paraná y sus afluentes.

Fuente: Google Eart. 2016 y reelaboración del equipo (Ricardo Lombardo y Ana María Attías).

Para que se entienda la magnitud del impacto de este conjunto de normas baste decir que entre el Tramo 1, desde el Sur de la provincia hasta la Capital de Corrientes en el Norte, y el Tramo 2, desde Paso de la Patria hasta Ituzaingó, las regulaciones impactan en una zona ribereña de aproximadamente $625 \mathrm{~km}$. Sólo están liberados unos $140 \mathrm{~km}$. Más aún, la zona de ribera que pertenece a Corrientes Capital, podría hacernos pensar que los pescadores comerciales pueden ejercer libremente su oficio. En los hechos, desde que se construyó la avenida costanera están inhabilitados para lanzar las redes $5 \mathrm{~km}$ aguas arriba y $5 \mathrm{~km}$ aguas abajo de la misma. Esto significa una gran desventaja en términos de desvinculación o alejamiento del mercado consumidor concentrado en la capital de la provincia. En oposición, en la zona costanera se habilitaron recientemente dos playas más, una de las cuales es privada.

\section{Pesca comercial, conflictos e indicadores de violencia}

A pesar de las restricciones que tienen los pescadores comerciales, ellos buscan mantenerse en su oficio.

Una de las mayores aspiraciones es dejar de sentirse ilegales, que los dejen pescar dignamente, que les den los permisos necesarios para hacer sus labores con tranquilidad. Manifiestan también que si habilitan de nuevo la zona que ahora está declarada reserva, aceptarían trabajar a reglamento.

Las restricciones sobre la cantidad de días, el tamaño de las piezas, sobre las especies y la altura de los ríos son factores comunes a todos los demás grupos de 
malloneros o comerciales. Uno de los problemas más acuciantes que afectan a comerciales de Bella Vista es la falta de puerto de desembarco, situación que los obliga a navegar de noche para evitar ser interceptados. Hay algunos pescadores que en esas situaciones se enfrentan a la autoridad argumentando la libertad de navegación. Pero no son los más numerosos. La siguiente anécdota relatada por uno de los entrevistados, ilustra una actitud propia de la violencia que emerge de la lucha por la supervivencia.

El otro día andando por allá me crucé con Fauna, y me dicen que me van a sacar la red, y yo le pregunto por qué. Me dicen que no se pude pescar en zona de reserva, yo le contesto que no estoy pescando, estoy navegando y para eso está el río. Ahora de que usted me quiera labrar un acta o secuestrarme la red porque no le gusta mi cara es otra cosa, pero no me va a sacar la red. Siempre van a estar con Prefectura, Prefectura está para avalar la actuación del funcionario, cualquier ataque físico que yo le pueda hacer entran ellos, sino no se pueden meter. Entonces les digo a ellos que se ocupen por la integridad física del funcionario, yo no le estoy atacando, estoy hablando nomás y con todo respeto. No estoy trabajando, estoy navegando. Les digo que, si me quieren sacar la red, vamos a casa, llamo un abogado y usted va a tener que explicar por qué me quiere sacar la red y aténganse a las consecuencias, yo no tengo nada que perder, usted va a perder el puesto. $Y$ ahí me dicen que trate de viajar por aquél lado, y yo le digo que voy a viajar por donde quiero, que porqué me van a prohibir. $Y$ ahí se fue. Tenemos que pelear por nuestros derechos. [Bella Vista, junio de 2014].

Para dar un cierre a este análisis vale explicitar que entendemos, como Pratesi (2014), que la violencia es polimorfa en tanto opera en los planos económico, político y jurídico y que se basa en relaciones asimétricas donde un elemento de la dupla tiene el poder de controlar y dominar al otro.

En el caso que nos ocupa podemos pensar que la coacción ejercida sobre este grupo de trabajadores del río configura un dispositivo tendiente a liberar el territorio donde desarrollan su actividad para ser explotado por otros sectores. En tanto se los despoja de derechos, de prestigio, de espacios y se minan sus relaciones sociales, se beneficia a otros grupos que acumulan estímulos económicos, legalidad, legitimidad y territorios para expandirse.

\section{Bibliografía}

Wellington Castellucci, Jr. (2007). Pescadores da Modernagem: cultura, trabalho e memoria em Tairu, BA (1960-1990). São Paulo: Annablume.

Hernández García, A. \& Sandoval Moreno, A. (2015). Agua y Tierra: Organización y reordenamiento de las tierras ganadas y actividades emergentes en el lago de Chapala, México (1904-2014). En Agua y Territorio, núm. 5, 111-120. http://revistaselectronicas.ujaen.es/index.php/atma/article/view/2538/2068

Piñeyro, N. y Serial, A. (2013). "Producir en las orillas. La pesca comercial en Chaco y Corrientes, Argentina". En VII Jornadas de Antropología Social Santiago Wallace. Organizadas por la Sección de Antropología Social del Instituto de Ciencias Antropológicas de la Facultad de Filosofía y Letras - UBA. Buenos Aires, 27, 28 y 29 de noviembre de 2013. Recuperado de http://www.aacademica.org/000-063/252 
Piñeyro, N., Attías, A. y Lombardo, R. (2015). La producción artesanal amenazada. La pesca con mallones en Chaco y Corrientes, Argentina, en la era de la economía global. En Castro, J. E. (Ed). Desigualdad, injusticia y cambio social: la suerte de las comunidades de pesca artesanal en América Latina. Cuadernos de Trabajo de la Red Waterlat- Gobacit, Serie Áreas Temáticas: Cuencas y Territorios Hidrosociales - SATCTH - vol. 2, núm. 4, ISSN 20564864 Versión digital y ISSN 2056-4856 Versión impresa (49-91). Newcastle, Upon Tyne, Reino Unido, Septiembre de 2015. http://waterlat.org/WPapers/WPSATCTH24.pdf

Pratesi, A. (2014). Relaciones violentas en el trabajo: más allá de la alienación. En RBSE Revista Brasileira da Sociología da Emoção, vol. 13, núm. 37, 104-112.

Saavedra Gallo, G. (2013). La Pesca Artesanal en las Encrucijadas de la Modernización. Usos, Apropiaciones y Conflictos en el Borde Costero del Sur de Chile. En Revista Andaluza de Antropología, núm. 4, 79-102. https://www.researchgate.net/publication/303065300 La pesca artesanal en I as encrucijadas de la modernizacion usos apropiaciones y conflictos en $e$ I borde costero del sur de Chile

\section{Documentos consultados}

Decreto Provincial Declaración de Reserva N 1970/89.

http://www.nuestromar.org/adobe/Marco regulario Pesca Continental Argentina.pdf

Disposición Dirección de Fauna №190/87.

http://www.nuestromar.org/adobe/Marco regulario Pesca Continental Argentina.pdf

Ley $N^{\circ} 4827 / 94$.

http://www.nuestromar.org/adobe/Marco regulario Pesca Continental Argentina.pdf

Decreto Nacional № 1034/52.

http://www.nuestromar.org/adobe/Marco regulario Pesca Continental Argentina.pdf

Decreto Provincial N³376/57.

http://www.nuestromar.org/adobe/Marco regulario Pesca Continental Argentina.pdf

Ley $N^{\circ} 3915 / 11$.

http://www.nuestromar.org/adobe/Marco regulario Pesca Continental Argentina.pdf

Sitio de Turismo Corrientes http://corrientesturismo.gob.ar/content/men\%C3\%BA-demapas-distancia-de-corrientes-ala-interior 